\title{
Formation of Fiber Fragments during Abrasion of Polyester Textiles
}

\author{
Yaping Cai, Denise M. Mitrano, Rudolf Hufenus, and Bernd Nowack*
}

Cite This: Environ. Sci. Technol. 2021, 55, 8001-8009

Read Online

ABSTRACT: Fiber fragments are one of the dominant types of microplastics in environmental samples, suggesting that synthetic textiles are a potential source of microplastics to the environment. Whereas the release of microplastics during washing of textiles is already well studied, much less is known about the release during abrasion processes. The abrasion of textiles may induce fibrillation of fibers and therefore result in the formation of much finer fiber fragments. The aim of this study was to investigate the influence of abrasion of synthetic textiles on the formation of microplastic fibers and fibrils. Fleece and interlock textile swatches made of polyester were abraded using abrasion tests with a Martindale tester. The microplastic fibers and fibrils formed during abrasion were extracted from the textiles and characterized in terms of number,

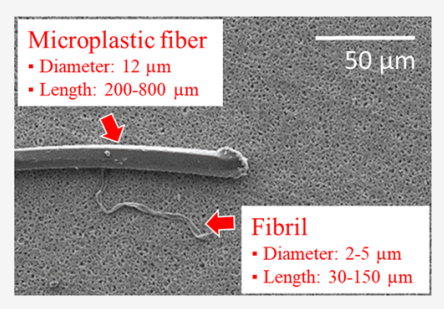
length, and diameter. The microplastic fibers demonstrated the same diameter than the fibers found in the textiles (fleece: $12.3 \mu \mathrm{m}$; interlock: $12.7 \mu \mathrm{m}$ ), while fibrils with a much smaller diameter (fleece: $2.4 \mu \mathrm{m}$; interlock: $4.9 \mu \mathrm{m}$ ) were also found. The number of fibrils formed during abrasion in both textiles was higher than the number of microplastic fibers. The majority of the extracted microplastic fibers had a length between 200 and $800 \mu \mathrm{m}$, while most fibrils were between 30 and $150 \mu \mathrm{m}$, forming two distinct fiber fragment morphologies. The number of microplastic fibers formed during abrasion was 5 to 30 times higher than the number of microplastic fibers that could be extracted from non-abraded samples. The number of fibrils increased after abrasion by more than a factor of $\mathbf{2 0 0}$ for both fabric types. The fibrils formed during abrasion have diameters that fall within the inhalable size for airborne particles. The potential release of fibrils into air during wear of textiles thus raises questions about the human exposure to these materials. Since the Martindale tester can simulate a daily application scenario of textiles over a prolonged period only in a limited way, future studies are needed to establish the correlation between the test results with a real-world scenario.

KEYWORDS: microplastic, textiles, release, fibers, fibrils, fragmentation

\section{INTRODUCTION}

Microplastic fibers (MPFs) are one of the dominant types of microplastics found in environmental samples, ${ }^{1-6}$ suggesting that synthetic textiles may be an important source of microplastics to the environment. For aquatic systems, one study estimated that about $35 \%$ of the global releases of microplastics into the ocean originated from washing of synthetic textiles. ${ }^{7}$ Similar conclusions were drawn for freshwaters by a recent modeling study, indicating that clothing ranked as the second largest source of all microplastics to Swiss freshwaters. ${ }^{8}$ It is estimated that about 0.17 to 0.28 million tons of MPFs reaches the aquatic system annually, 10 and the emission is expected to continue to grow in the future. ${ }^{10}$ The occurrence of microplastics has been detected in atmospheric fallout, ${ }^{3}$ rain, ${ }^{11}$ and household dust. ${ }^{4,12}$ The deposition rate of microplastics was estimated to range from 1586 to 11,130 particles $/ \mathrm{m}^{2} /$ day for the indoor environment, ${ }^{4,12}$ which is much higher than the rate obtained for the outdoor environment from remote US conservation areas (132 particles $/ \mathrm{m}^{2} /$ day $)^{11}$ or a European metropolitan region (137512 particles $/ \mathrm{m}^{2} /$ day). ${ }^{13}$ The size of the microplastics reported in the studies is usually between 11 and $5000 \mu \mathrm{m}$, while the majority of detected MPFs had a size below $500 \mu \mathrm{m} .{ }^{5,14} \mathrm{~A}$ study investigated the occurrence of MPFs in urban air in Beijing, where MPFs with a length below $20 \mu \mathrm{m}$ were reported to be the dominant type of airborne fibers. ${ }^{15}$ Since MPFs are commonly detected in air samples and the composition is consistent with those used in textiles, this raises the hypothesis that MPFs released from synthetic textiles are an important source of airborne microplastics. ${ }^{11,16}$

MPFs can be released from textiles to air during wearing of clothes. ${ }^{17}$ A recent study quantified MPF release into air from textiles, where garments made of different types of textiles were worn by volunteers and the MPFs released into air were collected on filter paper. ${ }^{17}$ The results showed that the MPF release ranged from 1 to 403 MPF per gram of textile with a length between 494 and $1034 \mu \mathrm{m}$, resembling the fibers released from textiles during washing. ${ }^{18}$ In addition, for most types of the textile samples, the release to air during wearing was approximately 10 times higher than the release to water during washing, highlighting the importance of wearing as a pathway of MPF release to the environment. ${ }^{18}$ Another study focused on the emission of fluorescent aerosol particles from

Received: January 29, 2021

Revised: May 5, 2021

Accepted: May 5, 2021

Published: June 1, 2021

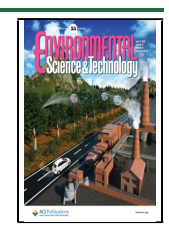


Table 1. Summary of the Experimental Conditions and the Different Control Samples Used ${ }^{a}$

\begin{tabular}{|c|c|c|c|c|c|}
\hline \multirow[b]{2}{*}{ experiment } & \multirow[b]{2}{*}{ aim } & \multirow[b]{2}{*}{ textile } & \multicolumn{2}{|r|}{ abrasion } & \multirow[b]{2}{*}{ extraction condition } \\
\hline & & & $\begin{array}{l}\text { put on the } \\
\text { Martindale } \\
\text { tester }\end{array}$ & abrasion conditions & \\
\hline $\begin{array}{l}\text { abrasion } \\
\text { experiment }\end{array}$ & investigate MPFs/fibrils formation during abrasion & $\begin{array}{l}\text { fleece, } \\
\text { interlock }\end{array}$ & yes & $12 \mathrm{kPa}, 5000$ rubs & $\begin{array}{l}1 \text { cycle }(45 \mathrm{~min}) \text { at } 40{ }^{\circ} \mathrm{C} \text {, } \\
\text { no steel balls, LAS solution }\end{array}$ \\
\hline $\begin{array}{l}\text { control- } \\
\text { abrasion }\end{array}$ & $\begin{array}{l}\text { determine the contamination (i.e., from the surroundings } \\
\text { and the operation of the Martindale tester) during } \\
\text { abrasion }\end{array}$ & $\begin{array}{l}\text { fleece, } \\
\text { interlock }\end{array}$ & yes & $\begin{array}{l}5000 \text { rubs, samples were } \\
\text { lifted to avoid abrasion }\end{array}$ & \\
\hline $\begin{array}{l}\text { control- } \\
\text { textile }\end{array}$ & $\begin{array}{l}\text { determine the baseline of extractable MPFs/fibrils in textile } \\
\text { samples }\end{array}$ & $\begin{array}{l}\text { fleece, } \\
\text { interlock }\end{array}$ & no & $\mathrm{N} / \mathrm{A}$ & \\
\hline $\begin{array}{l}\text { control- } \\
\text { extraction }\end{array}$ & $\begin{array}{l}\text { determine the contamination (i.e., from the LAS solution } \\
\text { and the steel vessels for washing) during extraction }\end{array}$ & none & no & $\mathrm{N} / \mathrm{A}$ & \\
\hline water wash & $\begin{array}{l}\text { investigate the influence of the addition of surfactant on } \\
\text { MPFs/fibrils extraction }\end{array}$ & fleece & yes & $12 \mathrm{kPa}, 5000$ rubs & $\begin{array}{l}1 \text { cycle }(45 \mathrm{~min}) \text { at } 40{ }^{\circ} \mathrm{C} \text {, } \\
\text { no steel balls, nanopure } \\
\text { water }\end{array}$ \\
\hline
\end{tabular}

volunteers wearing different garments made of polyester, cotton, or a mixture during specified activities in a closed chamber. ${ }^{19}$ The results showed that the mode size of the released fluorescent aerosol particles was between 3 and $5 \mu \mathrm{m}$ and volunteers wearing long clothing (i.e., long-sleeve shirts and pants) emitted more fluorescent aerosol particles than those wearing short clothing (i.e., t-shirts and shorts). ${ }^{19}$ Therefore, there is a possibility that microplastics with a much smaller size can be released from synthetic textiles to air during wearing.

It is still unknown how the abrasion of textiles after a period of use will affect the MPF formation. The MPFs observed in washing studies have a similar diameter to the original fibers in the textile. ${ }^{18,20}$ There is evidence that the application of repetitive shear stress on textile fibers can cause fiber fatigue, which subsequently leads to fiber failure. ${ }^{21}$ The yarn-on-yarn abrasion of polyester or nylon fibers under gentle conditions (leading to a long life in the test, e.g., failure after 35,000 cycles) can result in fibrillation, where axial fiber splitting occurs and much finer fibrils can be formed and worn away. ${ }^{21}$ This fibrillation does not only happen to synthetic fibers but can be also observed for natural fibers, such as cotton. ${ }^{21}$ The size of airborne fibers is relevant since it is generally acknowledged that particles with an aerodynamic diameter larger than 30-70 $\mu \mathrm{m}$ can quickly deposit. ${ }^{22}$ Particles with an aerodynamic diameter less than $10 \mu \mathrm{m}$ are often referred to as "inhalable particles". 22

The aim of this study was to investigate the influence of the abrasion of synthetic textiles on MPF and fibril formation (together designated as fiber fragments in the following). The abrasion experiment was conducted using a Martindale tester, which was originally designed to test the abrasion resistance of upholstery textiles. The MPFs and fibrils formed during abrasion were then extracted from the textile samples and deposited on a filter. A scanning electron microscope (SEM) was applied to characterize the fiber fragments on the filters, and then the fiber fragments were analyzed in terms of number, length, and diameter. The results from this work will not only lead to a better understanding of MPF and fibril formation during abrasion but also provide a new method for investigating MPF and fibril release during abrasion.

\section{MATERIALS AND METHODS}

2.1. Sample Preparation. Black-colored interlock and fleece polyester textiles were obtained directly from manu- facturers (Table S1 and Figure S1). The textiles were also used in previous studies ${ }^{18,20}$ to investigate the formation of MPFs during manufacturing and the release of MPFs during washing. The textile samples were cut using a laser cutter (tt-1300, Times technology) into circles with diameters of either $38 \mathrm{~mm}$ (specimen) or $140 \mathrm{~mm}$ (abradant) following the ISO standard $12947-2: 2016,{ }^{23}$ where the specimen is rubbed against the abradant during the abrasion test. The average weight for the fleece sample was $0.21 \mathrm{~g}$ (specimen) and $2.84 \mathrm{~g}$ (abradant), and for the interlock sample, it was $0.23 \mathrm{~g}$ (specimen) and 3.23 g (abradant).

A prewash step was performed to remove the majority of extractable MPFs generated during manufacturing ${ }^{20}$ as well as to reduce the interference by dusts and residues. All samples were prewashed three times with $150 \mathrm{~mL}$ of linear alkylbenzene sulfonic acid (LAS) solution $(0.75 \mathrm{~g} / \mathrm{L} \mathrm{pH} 9.2$ \pm 0.1 ) at $40{ }^{\circ} \mathrm{C}$ with a Gyrowash lab washing machine (James Heal, GyroWash model 1615) as described in previous washing studies. ${ }^{18,24}$ No steel balls were used during washing since the use of steel balls may result in higher abrasion. ${ }^{25}$ After prewashing, the textile samples were dried under aluminum foil at room temperature overnight. The change of sample weight before and after prewashing and drying was below $0.5 \%$.

2.2. Abrasion Experiments. The textile wearing process was simulated using a Martindale tester (4-station Martindale tester. SN-103/06/1049, James Heal) (Figure S2). This instrument is designed to test the abrasion resistance of the upholstery textile by rubbing textile samples against a standard surface under a specific pressure. ${ }^{23}$ The endpoint of the test is when the breakdown of textile samples can be determined, where the criteria of breakdown refer to "thread breakage" or "worn-off area" as indicated in the ISO standard 12947$2: 2016 .{ }^{23}$ The textile abrasion resistance can be evaluated by recording the number of rubs needed until the endpoint. The ISO standard aims at evaluating the quality of textile products, and thus, an extreme scenario is considered. Since our study was designed to simulate the daily use of textiles, the abrasion experiment was stopped after 5000 rubs. At this time, pilling was observed on the surface of the fabrics. Compared with complete thread breakage, the pilling phenomenon is commonly observed for polyester textiles under typical use conditions. $^{26}$ Some additional modifications were made to the ISO standard to collect MPFs and fibrils from the apparatus. First, the standard abradant was replaced with the same material as the specimen, thereby simulating a fabric-on-fabric 
rubbing situation. Second, the standard woven felt textile and the polyetherurethane foam material were not used as underlays to back the abradant and specimen, to avoid the input of additional fibers unrelated to the test textile into the system. Instead, rubber sheets (thickness of $1.5 \mathrm{~mm}$ ) were cut into the size of the specimen and abradant using scissors and were used to replace the standard overlays. The rubber sheet had a smooth surface from which fibers could be rinsed off more easily. Additionally, a metal ring made of stainless steel (inner diameter of $13.3 \mathrm{~cm}$, thickness of $1.3 \mathrm{~cm}$, and height of $4.8 \mathrm{~cm}$ ) was mounted on each station to avoid crosscontamination between samples and to collect the MPFs/ fibrils deposited to the apparatus (see Figure S3).

Before the abrasion test, the equipment was cleaned with liquid soap, water, and an air blower to remove any contamination. During each round of abrasion experiments, $12 \mathrm{kPa}$ pressure was randomly placed on three stations and the fourth one served as a control (noted as "control-abrasion" in Table 1). The setting for the control was the same as the abraded samples, except that the specimen was lifted from the abradant to avoid any abrasion. A white coat made of cotton and powderless nitrile gloves was worn throughout the experiment.

Since the system was not completely closed, we checked if MPFs or fibrils could escape from the system by placing double-sided carbon tape (PLANO G3347, diameter of 12 $\mathrm{mm}$ ) on the top, middle, and bottom of the three metal rings enclosing the abrasion samples, as shown in Figure S3. The tape was observed after the abrasion experiment under an SEM to assess if MPFs deposited on the metal rings.

2.3. MPF and Fibril Extraction. MPFs and fibrils were collected from both the apparatus (steel sample holder, ring, and rubber overlay) and extracted from the textile samples (abradant and specimen). LAS solution $(150 \mathrm{~mL})$ was used to rinse the apparatus, and the liquid was collected. MPFs and fibrils in the textile abradant and specimen were extracted separately with $150 \mathrm{~mL}$ of LAS solution using a GyroWash lab washing machine (James Heal, GyroWash model 1615) for 45 min at a temperature of $40{ }^{\circ} \mathrm{C}$. No steel balls were added during the washing. The samples were then squeezed to remove excess water. To better disperse the MPFs in the extraction liquid, the solution was sonicated for $3 \mathrm{~min}$ using an ultrasonic probe (Sonopuls HD, 2070; with probe VS $70 \mathrm{~T}$; power $70 \mathrm{~W}$; frequency $20 \mathrm{kHz}$; amplitude $25 \%$; pulse $1 \mathrm{~s}$ ). The sonication is unlikely to cause damage to the fibers and result in the generation of MPFs or fibrils as reported in a previous study. ${ }^{20}$ The solution was transferred using a pipette to a vacuum filtration system, and the solution was filtered onto a cellulose acetate membrane (diameter of $13 \mathrm{~mm}$, pore size of $0.45 \mu \mathrm{m}$; Sartorius $\mathrm{GmbH})$. Depending on the concentration of MPFs and fibrils determined in pretests, between 1 and $50 \mathrm{~mL}$ of extraction liquid was used as a subsample to avoid too much overlapping of fibers on the filter. The filtered volume for each sample can be found in Table S2. The filters were dried in a Petri dish with a cap at room temperature overnight.

To determine the contamination level during extraction, two blanks containing only LAS solution were randomly inserted into each extraction cycle (noted as "control-extraction" in Table 1). To assess the baseline of extractable MPFs from nonabraded samples, fleece and interlock textiles without abrasion were also extracted using the GyroWash lab washing machine. This set of experiments is indicated as "control-textile" in
Table 1. Therefore, together with "control-abrasion", controls at three different levels were conducted to assess the whole experimental workflow.

An additional experiment (i.e., "water wash" in Table 1) was performed using the fleece textile to investigate the extraction efficiency of MPFs and fibrils without a surfactant. The experimental procedure was the same as for the standard abrasion experiment, except that nanopure water was used to replace LAS solution as the liquid during extraction in the GyroWash lab washing machine.

2.4. Image Analysis. Filters were sputtered with a $7 \mathrm{~nm}$ layer of $\mathrm{Au} / \mathrm{Pd}$ using a high vacuum sputter coater (LEICA EM ACE600) before observation under SEM (7 kV, Quanta FEI 650). To determine the number and length of MPFs and fibrils, the filters were imaged at a magnification of 200 with a resolution of $1536 \times 1024$ pixels. A grid of $7 \times 10$ images (i.e., 70 photos total) were needed to cover the entire area of each filter, which was achieved by the automatic "mapping" function of the Quanta FEI 650. The 70 images were subsequently merged after manually checking and adjusting the position of each image. The complete image had a width of $13.248 \mathrm{~mm}$ with a resolution of $9669 \times 9132$ pixels $\left(\sim 166 \mathrm{~mm}^{2}\right)$. To determine the diameter of MPFs and fibrils, a grid of $4 \times 5$ images (i.e., 20 photos total) was taken at a higher magnification of $500 \times$. The final image had a width of $3.0673 \mathrm{~mm}$ with a resolution of $5582 \times 4601$ pixels $\left(\sim 8 \mathrm{~mm}^{2}\right)$. Examples of analyzed images are shown in Figures S4 and S5. The image analysis was carried out with ImageJ. ${ }^{27}$ The image captured all MPFs/fibrils in the subsample of the extraction liquid, and all MPFs and fibrils on the filters were labeled manually using a Microsoft surface tablet and a stylus pen. In total, 104 composite images were analyzed, where 2365 MPFs and fibrils were measured to evaluate the number and length, and 502 were used to assess the diameter.

Only fiber fragments with an aspect ratio greater than 3 were counted as fibers, as defined by the European Chemicals Agency. ${ }^{28}$ Two fiber morphologies were identified. The first type had a cylindrical shape and is referred to as "MPF" in the text. The second type of fibers had a "tape-like" shape and had a much smaller diameter. They are referred to as "fibrils". The detection limit for the length was 9-10 pixels at the magnification of $200 \times$, corresponding to $15 \mu \mathrm{m}$, while at the magnification of $500 \times$, the detection limit was $1 \mu \mathrm{m}$ (4-5 pixels). For the tape-shaped fibrils, the diameters were determined by measuring the widest part.

Additionally, SEM was used to characterize the surface of the textiles and the fibers protruding from the textile surface. The same sputtering method was applied as described above. The textile surface was imaged at a magnification of $100 \times$, while the fiber ends were characterized at a magnification of $2000 \times$. Fifty fiber ends were randomly imaged to estimate the percentage of truncated (i.e., solid ends) and fibrillated ends (i.e., fiber ends with axial fiber splitting at the end). Images at different magnifications (i.e., $500 \times, 2000 \times$, and $10,000 \times$ ) were taken by SEM to better characterize the fiber fragments. The percentage of truncated and fibrillated ends was estimated by randomly imaging 50 fiber ends at a magnification of $500 \times$.

2.5. Statistics. A one-way ANOVA test in IBM SPSS software (version 27) was used to determine the influence of the textile type on the number of extracted MPFs and fibrils. A nonparametric Kruskal-Wallis $(\mathrm{K}-\mathrm{W})$ one-way ANOVA test in IBM SPSS software (version 27) was used to compare the distribution of length and diameter in the following groups: 
(1) with abrasion and without abrasion; (2) different samples: apparatus, specimen, and abradant; and (3) with and without the addition of surfactant. A $p$ value of less than 0.05 was considered statistically significant.

\section{RESULTS}

3.1. Assessment of the Methodology. The ISO standard was not designed to capture released fibers, and therefore, various tests were performed to evaluate the suitability of our adapted methodology. The possibility that MPFs and fibrils escaped the enclosure around the abrasion stand was evaluated by analyzing double-sided carbon tape placed at different positions on the ring. On average, $4 \pm 4$ MPFs and $1 \pm 1$ fibrils were observed at the bottom of the ring, while 0 to $1 \mathrm{MPFs}$ or fibrils were observed at the upper side of the ring (Table S4). Therefore, it can be assumed that the majority of MPFs generated were contained and collected within this system and did not escape.

To optimize the extraction method, an experiment was carried out to assess the influence of the surfactant on the extraction of MPFs and fibrils after abrasion (Figure S6 and Tables S5 and S6). The number of MPFs and fibrils extracted from abraded textile samples with pure water was only $120 \pm$ 32 MPFs and $140 \pm 40$ fibrils per gram of textile, which was approximately 20 and 40 times lower than by extraction with LAS. There was no significant difference ( $p$ value of 0.52 , Table S7) observed between the length distribution of MPFs extracted with LAS (median: $290 \mu \mathrm{m}$ ) and water (median: 229 $\mu \mathrm{m})$. LAS tended to extract significantly shorter and thinner $(p$ value of 0.000 , Table S7) fibrils (length: $56 \mu \mathrm{m}$; diameter: 2.4 $\mu \mathrm{m}$ ) than water (length: $99 \mu \mathrm{m}$; diameter: $3.0 \mu \mathrm{m}$ ). Therefore, LAS was chosen to extract the MPFs and fibrils from textile samples.

MPFs and fibrils collected from the apparatus, specimen, and abradant were first separately analyzed for number, length, and diameter. To better compare the number of fibers extracted from different samples, the absolute values are shown in Figure S7, while the number normalized to the mass of the textiles are shown in Table S8. The absolute number of MPFs collected from the apparatus (fleece: $1700 \pm 843$; interlock: $2900 \pm 910$ ) was about more than one-thirds (fleece: 37\%; interlock: 64\%) compared to the one extracted from specimen and abradant. These results show that it is important to include the fibers deposited on the apparatus in the counting of fibers. In terms of the length distribution (Figure S7 and Table S9), the MPFs and fibrils collected from the apparatus usually demonstrated a significantly longer length ( $p$-value of 0.000 , Table S11) than those extracted from the specimen and abradant (e.g., fibrils from fleece: $453 \mu \mathrm{m}$ for apparatus, $57 \mu \mathrm{m}$ for specimen, and $53 \mu \mathrm{m}$ for abradant). There was no significant difference of the diameter between MPFs collected from apparatus, specimen, and abradant (Tables S10 and S11). The diameter of fibrils collected from different samples was also similar, except for interlock where significantly thicker fibrils ( $p$-value of 0.000 , Table S11) were found on the apparatus than in the textile samples (Figure S7 and Table S10). In the later analysis, the MPFs and fibrils collected from apparatus, specimen, and abradant were combined.

Several measurements were performed to assess the contamination and the reliability of the extraction and analytical procedures. To quantify the contamination during the extraction step, 14 blanks were assessed and indicated as "control-extraction" in Table 1 . The level of contamination was 0 to $2 \pm 1 \mathrm{MPFs}$ per $150 \mathrm{~mL}$ and 0 to $8 \pm 6$ fibrils per $150 \mathrm{~mL}$ depending on the set of experiments. The baseline of extractable MPFs or fibrils in textile samples was determined by analyzing the number of MPFs and fibrils extracted from textile samples without abrasion ("control-textile"), which was $410 \pm 230 \mathrm{MPFs}$ and $31 \pm 16$ fibrils per gram of textile for fleece and $69 \pm 36$ MPFs and fibrils below the detection limit for interlock. The detection limit considered here for fibrils was calculated as the mean of the blanks ("control-extraction") plus three times the standard deviation, which was 26 fibrils per gram of textile for interlock fabric. The baseline value shows the amount of MPFs or fibrils present in textile samples before abrasion. The MPFs and fibrils originated from this source accounted for about 20 and $0.51 \%$ for fleece and 3.1 and $0 \%$ for interlock. For "control-abrasion", the contamination of the apparatus (steel sample holder, ring, and rubber overlay) was 0 MPFs and $5 \pm 4$ fibrils during the whole abrasion period. The MPFs and fibrils found in the control-abrasion samples (abradant and specimen) were $590 \mathrm{MPF}$ and 160 fibrils per gram of textile for fleece and $87 \mathrm{MPFs}$ and 100 fibrils per gram of textile for interlock. The contamination during abrasion (the difference between the number of extracted MPFs or fibrils from "control-textile" and "control-abrasion") accounted for about 8 and $2 \%$ of those found in the abraded samples for fleece $(2100 \pm 930 \mathrm{MPF}$ per gram of textile; $6000 \pm 2700$ fibril per gram of textile) and about 0.8 and $2 \%$ for interlock $(2300 \pm 250 \mathrm{MPF}$ per gram of textile; $5300 \pm 640$ fibril per gram of textile). Therefore, MPFs and fibrils analyzed from the abraded samples were mainly formed during the abrasion process.

To determine the reliability of filtering to achieve the precise number of MPFs or fibrils measured, three aliquots from the same extraction solution were analyzed, as shown in Figure S8 and Table S12. The majority of the samples demonstrated a relative standard deviation below $15 \%$ (4-14\%), except for the release to apparatus for the fleece samples (MPF: 26\%; fibril: $44 \%)$. Considering the small size of the filter required for SEM analysis, which limited the number of analyzed MPFs (average of $23 \pm 15 \mathrm{MPFs}$ and $43 \pm 51$ fibrils per filter), the results showed that the subsamples were representative for the number of MPFs and fibrils, and the results obtained by the chosen workflow were reproducible. Moreover, all experiments were carried out in three replicates. The relative standard deviation of the MPF and fibril number ranged from 7 to $100 \%$ with an average of $38 \%$.

3.2. Characterization of Abraded Textiles and Fiber Fragments. The change of the surfaces after abrasion of the fleece textile samples can be observed from SEM images (Figure 1A,B). The surface of the textile without abrasion was fuzzy, while aggregations of fibers were observed on the surface of the abraded textile. When imaging the ends of fibers protruding from the surface of the textiles at a higher resolution, all randomly selected fibers from the non-abraded sample had truncated ends (Figure 1C). For the abraded samples (fleece, specimen), fibers with truncated ends also accounted for the majority of fibers observed (about 90\%), but approximately $10 \%$ of the fibers had fibrillated ends (Figure 1D), where a number of tiny fibrils protruded from the end of the fiber. These types of fiber ends were not observed in the non-abraded samples.

The MPFs and fibrils extracted from the samples were characterized by SEM (Figure 2). All MPFs extracted from the 


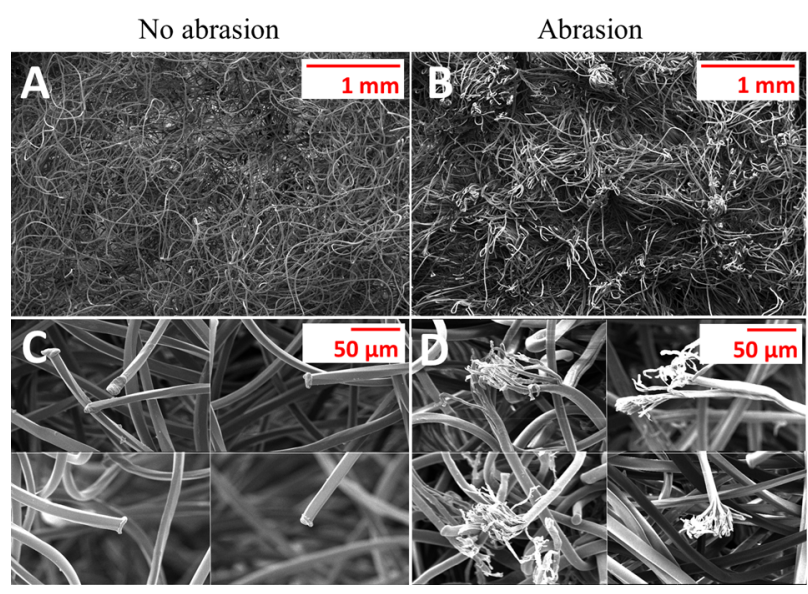

Figure 1. Characterization of the fibers of the surface of fleece. The images on the left show the non-abraded sample, and on the right side, the images show the abraded sample ( $12 \mathrm{kPa}, 5000$ rubs): (A) surface of fleece textile, non-abraded; (B) surface of fleece textile, abraded specimen; (C) solid fiber ends of fleece textile, non-abraded specimen; (D) fibrillated fiber ends of fleece textile, abraded specimen. SEM images were taken under $7 \mathrm{kV}$, the magnification for panels (A) and (B) was 100×, and for panels (C) and (D), it was 2000X. The scale bars in panels (C) and (D) apply to all the four small images. Similar images for the interlock sample are displayed in Figure S9.

non-abraded fleece textile demonstrated truncated ends, while fibrils were rarely observed (Figure 2A). For abraded samples, the number of extracted fibrils was much higher than the number of MPFs (Figure 2B), and both MPFs with solid ends and fibrillated ends could be observed. The MPFs with solid ends were identical to those found in non-abraded samples (Figure 2C) and accounted for the majority ( $>95 \%)$ of the fibers found from abraded fleece. A small portion $(<5 \%)$ of MPFs demonstrated fibrillated ends (Figure 2C), and this feature was the same compared to the fibrillated fiber ends observed on the textile surface (Figure 1D). Fibrils were further characterized at a higher magnification of $10,000 \times$ (Figure 2D). It can be seen that fibrils possessed a tape shape and had a much smaller diameter (about $2 \mu \mathrm{m}$ ) than the fibers (about $12 \mu \mathrm{m}$ ).

The number of MPFs and fibrils contained in the textiles after abrasion was quantified in the extraction solution. The numbers of MPFs extracted from fleece and interlock after abrasion were $2100 \pm 930$ and $2200 \pm 250 \mathrm{MPF}$ per gram of textile, which are 5 and 30 times higher than the numbers of MPFs extracted without abrasion (Figure 3A,D). In nonabraded samples, almost no fibrils were detected. In particular, for interlock, the number of fibrils was below the detection limit of 26 fibrils per gram for non-abraded samples and rose to $5300 \pm 650$ fibrils per gram of textile after abrasion. For fleece, the number of fibrils increased approximately 200 times from $31 \pm 16$ fibrils per gram of textile before abrasion to $6000 \pm$ 2700 fibrils per gram of textile after abrasion. The number of total MPFs and fibrils extracted from the non-abraded interlock sample was more than 5 times lower than the MPF extracted from the non-abraded fleece textile. However, the number of MPFs extracted from both types of textiles was not significantly different after abrasion ( $p$ value of 0.49 for MPF and 0.68 for fibril, Tables S13-S15).

The length distribution of the MPFs is displayed in Figure $3 \mathrm{~B}, \mathrm{E}$ as notched box plots. Overlapping notches of two boxes strongly indicate (95\% confidence) that the median of two distributions is not significantly different. ${ }^{29}$ For fleece, significantly longer MPFs were extracted from the abraded sample ( $p$ value of 0.000 , Table S20; median length of 290 $\mu \mathrm{m}$ ) than MPFs extracted from non-abraded samples (median length of $239 \mu \mathrm{m}$ ), which was opposite to the trend observed for fibrils (abraded: $56 \mu \mathrm{m}$; non-abraded: $143 \mu \mathrm{m}$; $p$ value of 0.000 , Table S20). For interlock, significantly shorter MPFs $(p$ value of 0.000 ; median length of $332 \mu \mathrm{m}$ ) were extracted from
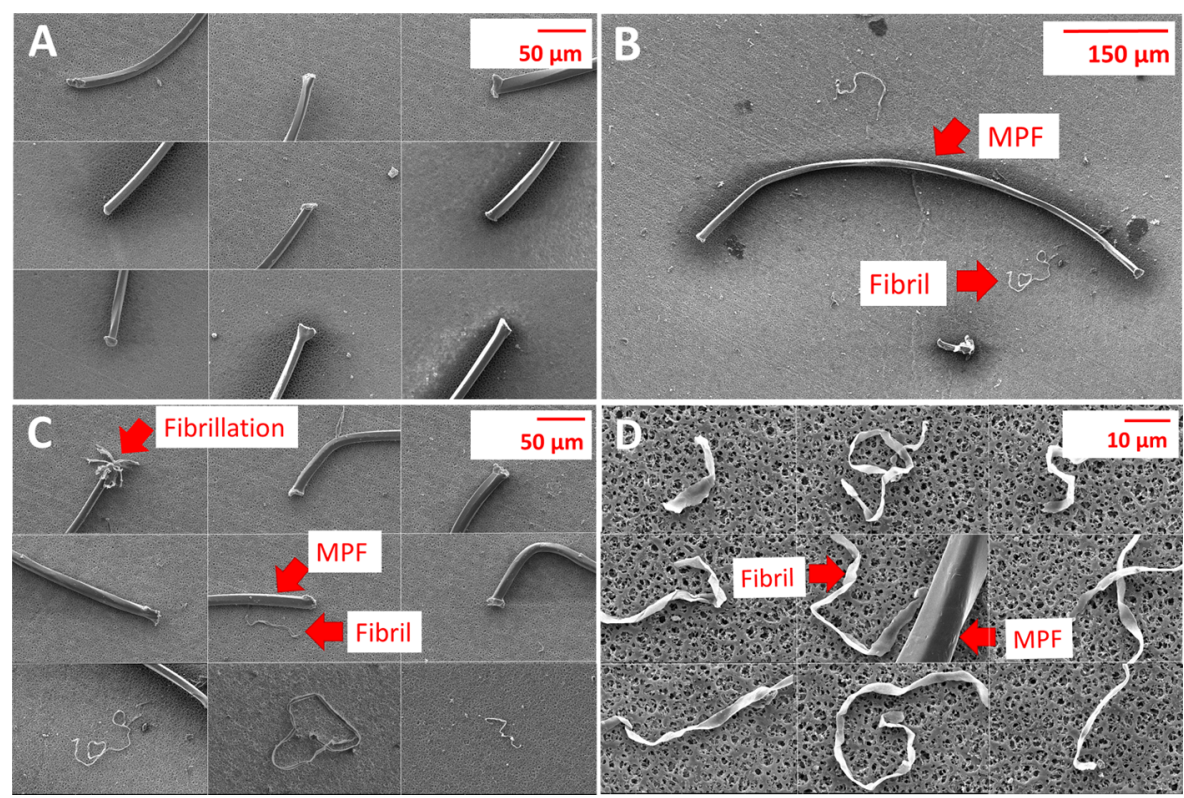

Figure 2. Characterization of MPFs and fibrils extracted from fleece: (A) MPFs extracted from non-abraded fleece textile at a magnification of $2000 \times$; (B) MPFs and fibrils extracted from abraded fleece textile at a magnification of $500 \times$; (C) MPFs and fibrils extracted from abraded fleece textile at a magnification of 2000×; (D) MPFs and fibrils extracted from abraded fleece textile at a magnification of 10,000×. The scale bars in panels (A), (C), and (D) apply to all the nine small images. Similar images for the interlock sample are displayed in Figure S10. 

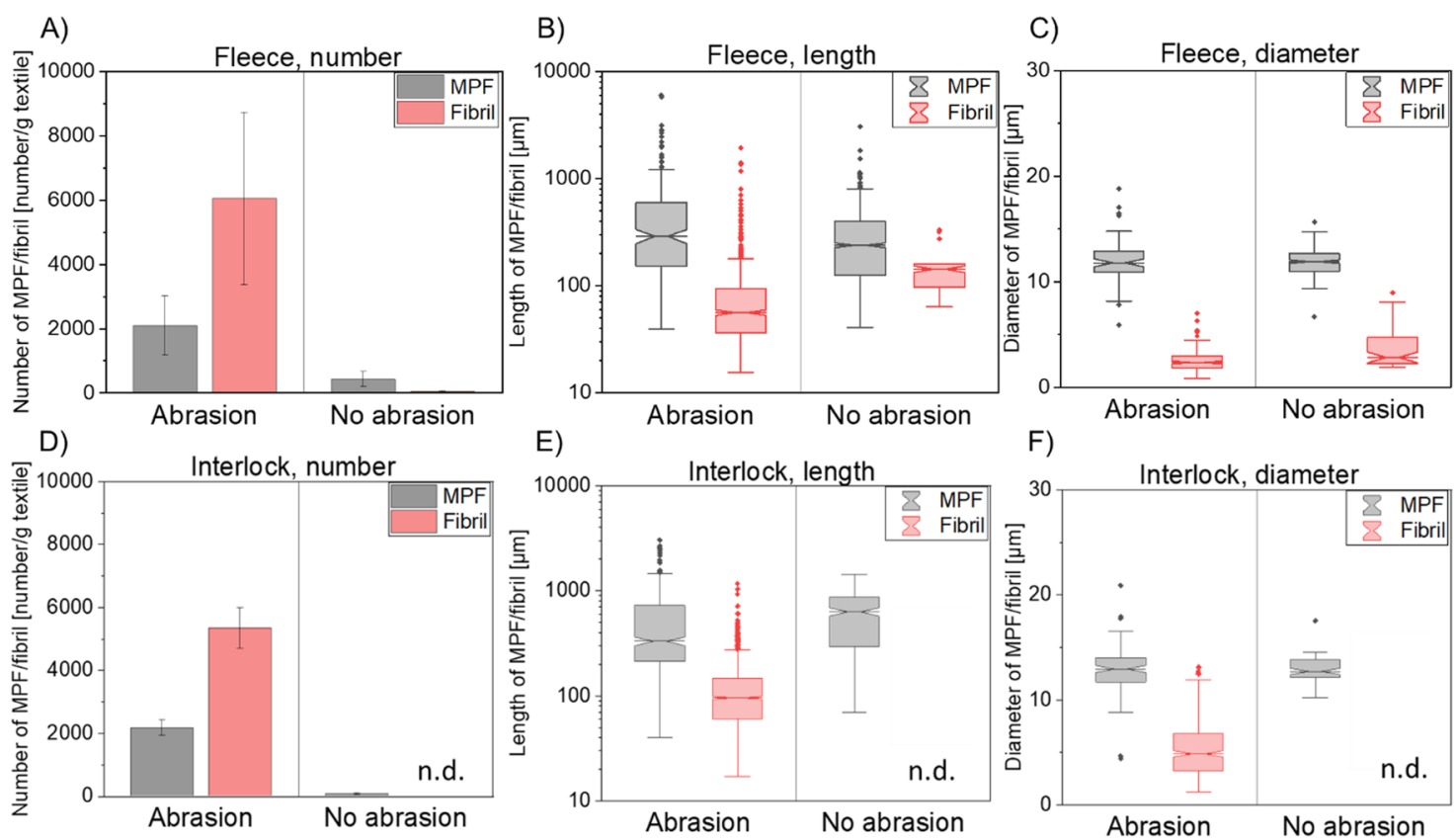

Figure 3. Number and distribution of length and diameter of MPFs and fibrils extracted from fleece and interlock textiles with and without abrasion. The length and diameter distribution presented here are a summation of triplicate experiments. For length distribution, the number of MPFs or fibrils counted per sample was between 13 and 835, with an average of 299. For the diameter distribution, the number of MPFs or fibrils plotted per sample was between 9 and 156 (Table S21), with an average of 67. 25th and 75th percentiles are represented by the boxes, and the median is indicated by a line. The number of extracted MPFs and fibrils is normalized to the mass of the textiles. n.d. refers to "not detected". The numerical values shown in the plots are given in Tables S16-S19 in the Supporting Information.

abraded samples than from non-abraded samples (median length of $632 \mu \mathrm{m}$ ). For both fleece and interlock, fibrils had a length that was 2 to 4 times smaller than that of the MPFs.

The average diameter of the MPFs extracted from abraded samples was 11.8 and $12.9 \mu \mathrm{m}$ for fleece and interlock, respectively (Figure 3C,F), which were similar to the ones observed within the textiles $(12.3 \pm 2.1$ for fleece and $12.7 \pm$ 1.5 for interlock), and there was also no significant difference between the MPFs extracted from abraded and non-abraded samples ( $p$ value of 0.40 for fleece and 0.08 for interlock, Table S20). Compared to MPFs, fibrils had much smaller diameters: $2.4 \mu \mathrm{m}$ for fleece and $4.9 \mu \mathrm{m}$ for interlock. Significantly thinner fibrils were found to be extracted from the abraded fleece (diameter of $2.4 \mu \mathrm{m}$ ) than from the non-abraded samples (diameter of $2.8 \mu \mathrm{m} ; p$ value of 0.000 ).

\section{DISCUSSION}

Microplastics in air are ubiquitous, and therefore, a careful examination of possible cross-contamination during sample handling is needed by carrying out various control experiments. In our experiments, the contamination during abrasion contributed a very small amount of MPFs and fibrils to those extracted from the abraded samples (MPF: 0.8-8\%; fibril: $2 \%$ ), suggesting that the interference from this source was under control. The contamination during extraction also contributed negligible amounts of fiber fragments (less than $0.1 \%)$ in the later analysis. Although the interference from production-inherited MPFs/fibrils in the textile samples could not be fully eliminated, the assessment shows that the majority of the MPFs (fleece: 71\%; interlock: 97\%) and fibrils (fleece: 96\%; interlock: $98 \%$ ) extracted from the abraded samples were formed during the abrasion process.

The abrasion process may affect the MPF extracted from synthetic textiles in two ways. First, previous work has shown that MPFs can already be contained in some textiles and likely originate from high-energy processes during textile manufacturing. ${ }^{18,20} \mathrm{MPFs}$ are found to be present in various textile products before the use phase. ${ }^{20}$ Processes that induce fiber breakage can be determined by characterizing the fiber end morphology, where high-energy cuts usually result in solid fiber ends without splits. ${ }^{21,30}$ The production-inherited MPFs often demonstrated solid ends similar to those generated by highenergy cut. ${ }^{18}$ In this case, the increase in MPFs extracted from textiles after abrasion is likely due to the mobilization of MPFs from the textile structure. Most of the MPFs (>95\%) extracted from abraded samples were found to have similar ends to those found from non-abraded samples and they are representative of fiber ends formed during high-energy cuts (e.g., scissors and knife). Therefore, the fraction of the MPFs with solid ends collected from the abraded samples was unlikely formed during the abrasion process but was liberated by the abrasion from within the yarns or the textile structure.

Compared with MPFs formed by high-energy cuts, abrasion often leads to fiber fatigue and fibrillation, resulting in the formation of much thinner fibrils. ${ }^{21}$ MPFs with fibrillated ends and fibrils with a shorter length and smaller diameter were extracted from the abraded textile samples, while the fibrillation of fibers was also observed at the textile surface after abrasion. A small number of fibrils were found in the nonabraded fleece, but for the interlock textile, the number of fibrils found for the non-abraded sample was below the detection limit. Therefore, it is unlikely that the washing process is responsible for the generation of fibrils because only a very limited amount of fibrils was found in non-abraded samples, which also went through the washing process. It cannot be excluded that a small amount of fibrils may have been formed during the textile manufacturing process and were already contained in the fabric similar to the MPF. Fleece 
textiles are known for their fuzzy texture, which is usually generated by a shearing process where surface fibers are cut $^{31,32}$ and this process may be responsible for the small amount of fibrils already contained in the fabric before abrasion.

The number of MPFs extracted from the non-abraded interlock sample was higher than that for fleece, but after abrasion, the number of MPFs extracted from both types of textile was about the same. Many studies investigated the factors affecting MPF release from different synthetic textiles during washing. ${ }^{18,24,26,33-43}$ MPF release from textiles has been reported to be influenced by textile properties including the type of yarns, surface treatment, and edge treatment. ${ }^{24,26,33-37,40-42}$ A recent study also investigated the influence of finishing treatment on fiber release from cotton textiles during washing, ${ }^{44}$ where the results show that the mechanical properties of fibers play an important role. Softer fabrics tended to form more fuzz and release more fibers. ${ }^{44}$ The application of a softener to cotton textiles can also reduce the fiber brittleness and increase abrasion resistance, resulting in the release of longer fibers. ${ }^{44}$ However, the influence of the softener on polyester textiles still needs to be investigated since polyester is hydrophobic, which may absorb much less softener than cotton. Further systematic research is also needed to understand how those textile properties can influence the MPF release after abrasion because only two different fabric types were used in this work.

There are several limitations of this study that need to be considered. First, the Martindale tester can only simulate a very limited daily application scenario of textiles such as sitting and moving with clothes on a chair and sofa over a prolonged period. Thus, the results obtained from the experiment describe the potential of MPFs and fibrils to be generated during several years of use. Further research needs to establish the influence of parameters such as the number of rubs and the level of pressure on fibril formation during abrasion. Second, in our abrasion tests, the specimen and the abradant were fixed on the sample holders and they were always in close contact with each other, which may hamper the release of fibers into the surrounding environment. To simulate a more realistic scenario, a chamber study needs to be conducted by volunteers wearing aged garments and establishing if under these conditions also a bimodal size distribution is obtained.

So far, the presence of fibrils and the bimodal size distribution of fiber fragments released from textiles have not been reported in textile release studies or in monitoring studies in the environment. The length of the fibrils formed during abrasion was shorter than the MPFs detected in aquatic and terrestrial systems, where the length of detected MPFs ranged from 500 to $1000 \mu \mathrm{m} .{ }^{45}$ Compared to microplastics in soil and water, the majority of MPFs found in air demonstrate a size below $500 \mu \mathrm{m} .{ }^{14}$ A study investigated the occurrence of MPFs in urban air in Beijing, where MPFs with a size below $20 \mu \mathrm{m}$ were reported to be the dominant types of airborne fibers. ${ }^{15}$ The size of MPFs in surface dust was found to be a bit longer than the airborne fibers with a major size between 25 and 200 $\mu \mathrm{m},{ }^{15}$ which is in accordance with the length of fibrils reported in our study.

A fraction of the airborne MPFs with a smaller size are believed to be inhalable ${ }^{46,47}$ and may be persistent in the lung and cause a biological response such as inflammation. ${ }^{48-50}$ Compared to the longer MPFs, the fibrils with a smaller size may raise more human exposure issues. The transport of particles in air is determined by their aerodynamic behavior, which depends on the aerodynamic diameter. ${ }^{51}$ For stiff fibers, the diameter or width of fibers plays the central role, controlling the aerodynamic diameter, and subsequently, the pulmonary deposition, ${ }^{52,53}$ while the length was reported to have little impact on fiber deposition in lungs. ${ }^{53}$ Although the fibrils discovered in our study have an average diameter between 2 and $5 \mu \mathrm{m}$, it is difficult to estimate the aerodynamic diameter of fibrils. The fibrils can demonstrate a high degree of curvature and flexibility compared to (comparatively more) stiff MPFs. Further studies are needed to investigate the aerodynamic behavior and the penetration of fibrils to the lower respiratory tract and the subsequent potential health hazards. It should be noted here that, if the mechanism of toxicity is merely a consequence of inhalation of fine particles, the fibrils generated from synthetic textiles would not necessarily be more hazardous than those from natural fibers, which may be easier to be broken down into smaller pieces than those with a synthetic origin. It is therefore necessary to also include natural fibers such as cotton into further studies about the release and hazard of fibrillated fibers.

In terms of the ecotoxicity of MPFs, a recent review by Bucci et al. ${ }^{54}$ has analyzed the data from 139 studies investigating the ecotoxicological effects by plastic debris, and the results show that the shape of the particles is an important factor affecting the effects of microplastics. The observed effects ranged from no effects, ${ }^{55}$ effects at the cellular level (e.g., mucosal damage, inflammation, and metabolism disruption), ${ }^{56}$ and effects at the sub- and organism level (e.g., longer egestion times, reduced growth, and mortality). ${ }^{57-59}$ Some studies compared the toxicity of MPFs with microplastics with other shapes, and MPFs were found to be more toxic to shrimps (Palaemonetes pugio $)^{58}$ and a crustaceans (Hyalella azteca) ${ }^{57}$ than spheres and fragments, respectively. Fibers were also found to cause a higher degree of intestinal damage after ingestion by zebra fish than beads and fragments. ${ }^{56}$ The size of MPFs is also reported to play a role in the toxicological effects to organisms, where studies show that longer fibers are more toxic to shrimps ${ }^{58}$ and zebra fish. ${ }^{60}$ However, the fibers used in those studies had a diameter of $20 \mu \mathrm{m}$, which is much larger than the diameter of fibrils found in this study. Microplastics with a smaller size are reported to exert more likely effects ${ }^{54}$ since smaller particles are more bioavailable ${ }^{61}$ and easier to translocate across the gut/extracellular barrier. ${ }^{62}$ Therefore, the fibrils with a much smaller size and diameter may also have different ecotoxicological effects on aquatic and terrestrial organisms than MPFs, which need to be carefully assessed in the future.

\section{ASSOCIATED CONTENT}

\section{Supporting Information}

The Supporting Information is available free of charge at https://pubs.acs.org/doi/10.1021/acs.est.1c00650.

Details about the materials and the experimental methods; results of the assessment of the methodology; tables with numerical values shown in the plots (PDF)

\section{AUTHOR INFORMATION}

\section{Corresponding Author}

Bernd Nowack - Technology and Society Laboratory, Empa Swiss Federal Laboratories for Materials Science and Technology, St. Gallen 9014, Switzerland; ㅇorcid.org/ 
0000-0002-5676-112X; Phone: +41 58765 7692;

Email: nowack@empa.ch

\section{Authors}

Yaping Cai - Technology and Society Laboratory, Empa Swiss Federal Laboratories for Materials Science and Technology, St. Gallen 9014, Switzerland; (1) orcid.org/ 0000-0001-8207-8132

Denise M. Mitrano - Department of Environmental Systems Science, ETH Zürich, Zürich 8092, Switzerland; Process Engineering Department, Eawag-Swiss Federal Institute of Aquatic Science and Technology, Dübendorf 8600, Switzerland; (1) orcid.org/0000-0001-8030-6066 Rudolf Hufenus - Laboratory for Advanced Fibers, EmpaSwiss Federal Laboratories for Materials Science and Technology, St. Gallen 9014, Switzerland

Complete contact information is available at: https://pubs.acs.org/10.1021/acs.est.1c00650

\section{Notes}

The authors declare no competing financial interest.

\section{ACKNOWLEDGMENTS}

We would like to thank Manfred Heuberger from Empa for the valuable feedback and discussion. This research was partially supported by funds from the Zürcher Stiftung für Textilforschung. D.M.M. was supported by the Swiss National Science Foundation grant nos. PZP002_168105 and PCEFP2_186856.

\section{REFERENCES}

(1) Anderson, P. J.; Warrack, S.; Langen, V.; Challis, J. K.; Hanson, M. L.; Rennie, M. D. Microplastic contamination in Lake Winnipeg, Canada. Environ. Pollut. 2017, 225, 223-231.

(2) Su, L.; Xue, Y.; Li, L.; Yang, D.; Kolandhasamy, P.; Li, D.; Shi, H. Microplastics in Taihu Lake, China. Environ. Pollut. 2016, 216, 711719.

(3) Dris, R.; Gasperi, J.; Saad, M.; Mirande, C.; Tassin, B. Synthetic fibers in atmospheric fallout: A source of microplastics in the environment? Mar. Pollut. Bull. 2016, 104, 290-293.

(4) Dris, R.; Gasperi, J.; Mirande, C.; Mandin, C.; Guerrouache, M.; Langlois, V.; Tassin, B. A first overview of textile fibers, including microplastics, in indoor and outdoor environments. Environ. Pollut. 2017, 221, 453-458.

(5) Wang, C.; Zhao, J.; Xing, B. Environmental source, fate, and toxicity of microplastics. J. Hazard. Mater. 2021, 407, 124357.

(6) Chan, C.; Park, C.; Chan, K. M.; Mak, D. C.; Fang, J. K. H.; Mitrano, D. M. Microplastic fibre releases from industrial wastewater effluent: a textile wet processing mill in China. Environ. Chem. 2021, DOI: $10.1071 /$ en20143.

(7) Boucher, J.; Friot, D. Primary Microplastics in the Oceans: a Global Evaluation of Sources; IUCN: Gland, Switzerland, 2017; 43.

(8) Kawecki, D.; Nowack, B. Polymer-Specific Modeling of the Environmental Emissions of Seven Commodity Plastics As Macroand Microplastics. Environ. Sci. Technol. 2019, 53, 9664-9676.

(9) Belzagui, F.; Gutiérrez-Bouzán, C.; Álvarez-Sánchez, A.; Vilaseca, M. Textile microfibers reaching aquatic environments: A new estimation approach. Environ. Pollut. 2020, 265, 114889.

(10) Gavigan, J.; Kefela, T.; Macadam-Somer, I.; Suh, S.; Geyer, R. Synthetic microfiber emissions to land rival those to waterbodies and are growing. PLoS One 2020, 15, No. e0237839.

(11) Brahney, J.; Hallerud, M.; Heim, E.; Hahnenberger, M.; Sukumaran, S. Plastic rain in protected areas of the United States. Science 2020, 368, 1257-1260.

(12) Catarino, A. I.; Macchia, V.; Sanderson, W. G.; Thompson, R. C.; Henry, T. B. Low levels of microplastics (MP) in wild mussels indicate that MP ingestion by humans is minimal compared to exposure via household fibres fallout during a meal. Environ. Pollut. 2018, 237, 675-684.

(13) Klein, M.; Fischer, E. K. Microplastic abundance in atmospheric deposition within the Metropolitan area of Hamburg, Germany. Sci. Total Environ. 2019, 685, 96-103.

(14) Zhang, Y.; Kang, S.; Allen, S.; Allen, D.; Gao, T.; Sillanpää, M. Atmospheric microplastics: A review on current status and perspectives. Earth-Science Reviews 2020, 203, 103118.

(15) Li, Y.; Shao, L.; Wang, W.; Zhang, M.; Feng, X.; Li, W.; Zhang, D. Airborne fiber particles: Types, size and concentration observed in Beijing. Sci. Total Environ. 2020, 705, 135967.

(16) Chen, G.; Feng, Q.; Wang, J. Mini-review of microplastics in the atmosphere and their risks to humans. Sci. Total Environ. 2020, 703, 135504.

(17) De Falco, F.; Cocca, M.; Avella, M.; Thompson, R. C. Microfiber Release to Water, Via Laundering, and to Air, via Everyday Use: A Comparison between Polyester Clothing with Differing Textile Parameters. Environ. Sci. Technol. 2020, 54, 3288-3296.

(18) Cai, Y.; Yang, T.; Mitrano, D. M.; Heuberger, M.; Hufenus, R.; Nowack, B. Systematic Study of Microplastic Fiber Release from 12 Different Polyester Textiles during Washing. Environ. Sci. Technol. 2020, 54, 4847-4855.

(19) Yang, S.; Bekö, G.; Wargocki, P.; Williams, J.; Licina, D. Human Emissions of Size-Resolved Fluorescent Aerosol Particles: Influence of Personal and Environmental Factors. Environ. Sci. Technol. 2021, 55, 509-518.

(20) Cai, Y.; Mitrano, D. M.; Heuberger, M.; Hufenus, R.; Nowack, $\mathrm{B}$. The origin of microplastic fiber in polyester textiles: The textile production process matters. J. Cleaner Prod. 2020, 267, 121970.

(21) Hearle, J. W.; Lomas, B.; Cooke, W. D. Atlas of fibre fracture and damage to textiles; Elsevier: 1998.

(22) Englert, N. Fine particles and human health-a review of epidemiological studies. Toxicol. Lett. 2004, 149, 235-242.

(23) ISO Standard 12947-2:2016. Textiles - Determination of the abrasion resistance of fabrics by the Martindale method - Part 2: Determination of specimen breakdown; ISO: 2016.

(24) Hernandez, E.; Nowack, B.; Mitrano, D. M. Polyester Textiles as a Source of Microplastics from Households: A Mechanistic Study to Understand Microfiber Release During Washing. Environ. Sci. Technol. 2017, 51, 7036-7046.

(25) Zambrano, M. C.; Pawlak, J. J.; Daystar, J.; Ankeny, M.; Cheng, J. J.; Venditti, R. A. Microfibers generated from the laundering of cotton, rayon and polyester based fabrics and their aquatic biodegradation. Mar. Pollut. Bull. 2019, 142, 394-407.

(26) Napper, I. E.; Thompson, R. C. Release of synthetic microplastic plastic fibres from domestic washing machines: Effects of fabric type and washing conditions. Mar. Pollut. Bull. 2016, 112, $39-45$.

(27) Abràmoff, M. D.; Magalhães, P. J.; Ram, S. J. Image processing with ImageJ. Biophotonics Int. 1998, 11, 36-42.

(28) ECHA Opinion on an Annex XV dossier proposing restrictions on intentionally-added microplastics; ECHA: 2020.

(29) Chambers, J. M.; Cleveland, W. S.; Kleiner, B.; Tukey, P. A. Graphical Methods for Data Analysis; Wadsworth International Group: 1983.

(30) Morton, W. E.; Hearle, J. W. S. Physical Properties of Textile Fibres; Fourth Ed.; Woodhead Publishing Series in Textiles, Elsevier: $2008,509-558$.

(31) Choudhury, A. K. R. Principles of textile finishing; Woodhead Publishing: 2017.

(32) Gries, T.; Veit, D.; Wulfhorst, B. Textile technology: an introduction; Carl Hanser Verlag GmbH Co KG: 2015.

(33) Carney Almroth, B. M.; Åström, L.; Roslund, S.; Petersson, H.; Johansson, M.; Persson, N.-K. Quantifying shedding of synthetic fibers from textiles; a source of microplastics released into the environment. Environ. Sci. Pollut. Res. 2018, 25, 1191-1199.

(34) de Falco, F.; Gullo, M. P.; Gentile, G.; di Pace, E.; Cocca, M.; Gelabert, L.; Brouta-Agnésa, M.; Rovira, A.; Escudero, R.; Villalba, R.; Mossotti, R.; Montarsolo, A.; Gavignano, S.; Tonin, C.; Avella, M. 
Evaluation of microplastic release caused by textile washing processes of synthetic fabrics. Environ. Pollut. 2018, 236, 916-925.

(35) Hartline, N. L.; Bruce, N. J.; Karba, S. N.; Ruff, E. O.; Sonar, S. U.; Holden, P. A. Microfiber Masses Recovered from Conventional Machine Washing of New or Aged Garments. Environ. Sci. Technol. 2016, 50, 11532-11538.

(36) Henry, B.; Laitala, K.; Klepp, I. G. Microfibres from apparel and home textiles: Prospects for including microplastics in environmental sustainability assessment. Sci. Total Environ. 2019, 652, 483-494.

(37) Jönsson, C.; Levenstam Arturin, O.; Hanning, A. C.; Landin, R.; Holmström, E.; Roos, S. Microplastics Shedding from TextilesDeveloping Analytical Method for Measurement of Shed Material Representing Release during Domestic Washing. Sustainability 2018, 10, 2457.

(38) McIlwraith, H. K.; Lin, J.; Erdle, L. M.; Mallos, N.; Diamond, M. L.; Rochman, C. M. Capturing microfibers-marketed technologies reduce microfiber emissions from washing machines. Mar. Pollut. Bull. 2019, 139, 40-45.

(39) Pirc, U.; Vidmar, M.; Mozer, A.; Kržan, A. Emissions of microplastic fibers from microfiber fleece during domestic washing. Environ. Sci. Pollut. Res. 2016, 23, 22206-22211.

(40) Sillanpää, M.; Sainio, P. Release of polyester and cotton fibers from textiles in machine washings. Environ. Sci. Pollut. Res. 2017, 24, 19313-19321.

(41) Belzagui, F.; Crespi, M.; Álvarez, A.; Gutiérrez-Bouzán, C.; Vilaseca, M. Microplastics' emissions: Microfibers' detachment from textile garments. Environ. Pollut. 2019, 248, 1028-1035.

(42) De Falco, F.; Di Pace, E.; Cocca, M.; Avella, M. The contribution of washing processes of synthetic clothes to microplastic pollution. Sci. Rep. 2019, 9, 6633.

(43) Kelly, M. R.; Lant, N. J.; Kurr, M.; Burgess, J. G. Importance of Water-Volume on the Release of Microplastic Fibers from Laundry. Environ. Sci. Technol. 2019, 53, 11735-11744.

(44) Zambrano, M. C.; Pawlak, J. J.; Daystar, J.; Ankeny, M.; Venditti, R. A. Impact of dyes and finishes on the microfibers released on the laundering of cotton knitted fabrics. Environ. Pollut. 2021, 272, 115998.

(45) Gago, J.; Carretero, O.; Filgueiras, A. V.; Viñas, L. Synthetic microfibers in the marine environment: A review on their occurrence in seawater and sediments. Mar. Pollut. Bull. 2018, 127, 365-376.

(46) Vianello, A.; Jensen, R. L.; Liu, L.; Vollertsen, J. Simulating human exposure to indoor airborne microplastics using a Breathing Thermal Manikin. Sci. Rep. 2019, 9, 1-11.

(47) Pauly, J. L.; Stegmeier, S. J.; Allaart, H. A.; Cheney, R. T.; Zhang, P. J.; Mayer, A. G.; Streck, R. J. Inhaled cellulosic and plastic fibers found in human lung tissue. Cancer Epidemiol. Biomark. Prev. 1998, 7, 419-428.

(48) Gasperi, J.; Wright, S. L.; Dris, R.; Collard, F.; Mandin, C.; Guerrouache, M.; Langlois, V.; Kelly, F. J.; Tassin, B. Microplastics in air: are we breathing it in? Curr. Opin. Environ. Sci. Health 2018, 1, 15.

(49) Prata, J. C. Airborne microplastics: Consequences to human health? Environ. Pollut. 2018, 234, 115-126.

(50) van Dijk, F.; Song, S. S.; van Eck, G.; Wu, X. H.; Bos, S.; Boom, D.; Kooter, I.; Spierings, D. C. N.; Wardenaar, R.; Cole, M. Inhalable textile microplastic fibers impair airway epithelial growth. bioRxiv 2021.

(51) Kreyling, W. G.; Möller, W.; Semmler-Behnke, M.; Oberdörster, G.; Donaldson, K.; Borm, P. Particle dosimetry: deposition and clearance from the respiratory tract and translocation towards extrapulmonary sites. Particle toxicology; CRC Press: 2007.

(52) Donaldson, K. The inhalation toxicology of p-aramid fibrils. Crit. Rev. Toxicol. 2009, 39, 487-500.

(53) Sturm, R. A theoretical approach to the deposition of cancerinducing asbestos fibers in the human respiratory tract. Lung Cancer 2009, 2, 1-11.

(54) Bucci, K.; Tulio, M.; Rochman, C. What is known and unknown about the effects of plastic pollution: A meta-analysis and systematic review. Ecol. Appl. 2020, 30, e02044.
(55) Jemec, A.; Horvat, P.; Kunej, U.; Bele, M.; Kržan, A. Uptake and effects of microplastic textile fibers on freshwater crustacean Daphnia magna. Environ. Pollut. 2016, 219, 201-209.

(56) Qiao, R.; Deng, Y.; Zhang, S.; Wolosker, M. B.; Zhu, Q.; Ren, H.; Zhang, Y. Accumulation of different shapes of microplastics initiates intestinal injury and gut microbiota dysbiosis in the gut of zebrafish. Chemosphere 2019, 236, 124334.

(57) Au, S. Y.; Bruce, T. F.; Bridges, W. C.; Klaine, S. J. Responses of Hyalella azteca to acute and chronic microplastic exposures. Environ. Toxicol. Chem. 2015, 34, 2564-2572.

(58) Gray, A. D.; Weinstein, J. E. Size- and shape-dependent effects of microplastic particles on adult daggerblade grass shrimp (Palaemonetes pugio). Environ. Toxicol. Chem. 2017, 36, 3074-3080.

(59) Ziajahromi, S.; Kumar, A.; Neale, P. A.; Leusch, F. D. L. Impact of Microplastic Beads and Fibers on Waterflea (Ceriodaphnia dubia) Survival, Growth, and Reproduction: Implications of Single and Mixture Exposures. Environ. Sci. Technol. 2017, 51, 13397-13406.

(60) Zhao, Y.; Qiao, R.; Zhang, S.; Wang, G. Metabolomic profiling reveals the intestinal toxicity of different length of microplastic fibers on zebrafish (Danio rerio). J. Hazard. Mater. 2021, 403, 123663.

(61) Jeong, C.-B.; Kang, H.-M.; Lee, M.-C.; Kim, D.-H.; Han, J.; Hwang, D.-S.; Souissi, S.; Lee, S.-J.; Shin, K.-H.; Park, H. G.; Lee, J.-S. Adverse effects of microplastics and oxidative stress-induced MAPK/ Nrf2 pathway-mediated defense mechanisms in the marine copepod Paracyclopina nana. Sci. Rep. 2017, 7, 1-11.

(62) Browne, M. A.; Dissanayake, A.; Galloway, T. S.; Lowe, D. M.; Thompson, R. C. Ingested microscopic plastic translocates to the circulatory system of the mussel, Mytilus edulis (L.). Environ. Sci. Technol. 2008, 42, 5026-5031. 